\title{
Short-pulse high-intensity excimer lasers - A powerful tool for the generation of coherent VUV and XUV radiation
}

\author{
B. Wellegehausen, K. Mossavi, A. Egbert, B. N. Chichkov, H. Welling \\ Institut für Quantenoptik, Universität Hannover, Welfengarten 1, 30167 Hannover \\ Received: 9 May 1996
}

\begin{abstract}
Different approaches for the generation of coherent VUV and XUV radiation with a $400 \mathrm{fs} \mathrm{KrF}$ excimer-laser system are studied. In nonlinear optical experiments it is shown that four-wave difference-frequency mixing in $\mathrm{Xe}$, using a near two-photon resonance with the $\mathrm{KrF}$ laser radiation, is well suited for the generation of tunable VUV radiation in the range 130-200 nm. Conversion efficiencies of $2 \%$ and output energies up to $260 \mu \mathrm{J}$ have been demonstrated. Further prospects to achieve $\mathrm{mJ}$ energies are discussed. Using this VUV source and the $\mathrm{KrF}$ laser, powerful XUV radiation can be generated by different low-order frequency mixing processes. In first experiments on this subject, direct frequency tripling of the $\mathrm{KrF}$ laser pulse has resulted in $14 \mu \mathrm{J} \mathrm{XUV}$ radiation at $83 \mathrm{~nm}$.

For the realization of soft-X-ray lasers, specific advantages of short-pulse $\mathrm{KrF}$ drivers are discussed. Novel scenarios based on a hybrid $\mathrm{KrF} / \mathrm{Ti}$ : sapphire laser system and multiphoton resonant excitation are considered.
\end{abstract}

PACS: $42.65 . \mathrm{Ky} ; 52.40 . \mathrm{Db}$

The development of practical coherent radiation sources operating at short wavelengths, in the VUV (below $200 \mathrm{~nm}$ ), XUV (below $100 \mathrm{~nm}$ ) and especially in the X-ray region (below $30 \mathrm{~nm}$ ), is among the most important and long-standing problems in laser physics. For example, speculations and proposals on the possibility of X-ray lasing have begun immediately after the invention of lasers, but the first successful demonstrations of X-ray lasers (at about $20 \mathrm{~nm}$ ) have only been reported in 1985 $[1,2]$. On the other hand, using methods of nonlinear optics, the generation of coherent radiation at $38 \mathrm{~nm}$ has already been observed in 1977, by successive frequency up-conversion of $1.06 \mu \mathrm{m} \mathrm{Nd:YAG}$ laser radiation in

Dedicated to Prof. F. P. Schäfer on the occasion of his 65 th birthday. nonlinear crystals and gases [3]. In these experiments ns laser pulses with energies of the order of $\mathrm{kJ}$ (X-ray lasers) and $30 \mathrm{ps}, 60 \mathrm{~mJ}$ laser pulses (nonlinear optics) have been used.

A key parameter for the efficient generation of shortwavelength coherent radiation is the laser intensity. For high-order nonlinear processes and for the creation of highly ionized non-equilibrium plasmas, very high laser intensities, above $10^{15} \mathrm{~W} / \mathrm{cm}^{2}$, are necessary. Due to the recent progress in laser technology, such and higher intensities can be easily realized with modern compact shortpulse laser systems. In these high-power laser systems, low-intensity short pulses are generated by a suitable oscillator and then amplified in one or more amplification stages. Design of the oscillator and amplifier systems depends on the laser material. Short-pulse high-power laser systems may be realized with solid and gaseous laser materials. In commercial systems, Ti:sapphire and the $\mathrm{KrF}$ excimer are most frequently used. Some properties of these laser materials for short-pulse high-power laser applications are summarized in Table 1 .

With Ti:sapphire crystals, both the generation of short pulses (typically by Kerr lens mode locking of $\mathrm{cw}$ argon ion-laser-pumped systems [4]) and their amplification in regenerative or multipass amplifiers (pumped by frequency-doubled Nd:YAG lasers) are possible. The bandwidth of the lasing transition allows to produce pulses as short as $10 \mathrm{fs}$. Due to the large saturation fluence, an energy of about $1 \mathrm{~J}$ can be extracted from a $1 \mathrm{~cm}^{3}$ crystal. However, the high Kerr constant of the material does not allow direct amplification of short pulses to such energies. This is performed with the Chirped Pulse Amplification (CPA) technique [5].

$\mathrm{KrF}$ excimer-laser systems can amplify pulses down to about $100 \mathrm{fs}$, but since the mode-coupling technique cannot be applied, they are not suited for the generation of these pulses. Short pulses have to be produced by other systems. Typically, frequency-doubled radiation from a short-pulse dye laser operating at $496 \mathrm{~nm}$ is used for this purpose. Because the nonlinearities in the $\mathrm{KrF}$ system are low, the short pulses can directly be amplified without the CPA technique. Due to the much smaller saturation 
Table 1. Parameters for shortpulse laser/amplifier systems

\begin{tabular}{lll}
\hline Laser system & $\mathrm{KrF}$ & Ti:sapphire \\
\hline Storage time & $7 \mathrm{~ns}$ & $3 \mu \mathrm{s}$ \\
Saturation fluence & $2 \mathrm{~mJ} / \mathrm{cm}^{2}$ & $1 \mathrm{~J} / \mathrm{cm}^{2}$ \\
Small signal gain coefficient & $\simeq 0.2 \mathrm{~cm}^{-1}$ & $\simeq 1.4 \mathrm{~cm}^{-1}$ \\
Short-pulse generation & External & Kerr lens mode locking \\
Short-pulse limit & $\simeq 100 \mathrm{fs}$ & $\simeq 10 \mathrm{fs}$ \\
Nonlinear refractive index & - & $3.5 \times 10^{-16} \mathrm{~cm}^{2} / \mathrm{W}$ \\
Short-pulse amplification & Direct & $\mathrm{CPA}$ \\
Energy & $3 \mathrm{~J}^{\mathrm{b}}$ & $>1 \mathrm{~J}^{\mathrm{c}}$ \\
Peak power & $10 \mathrm{TW}^{\mathrm{b}}$ & $100 \mathrm{TW}^{\mathrm{c}}$ \\
Focused intensity & $>10^{18} \mathrm{~W} / \mathrm{cm}^{2}$ & $>10^{18} \mathrm{~W} / \mathrm{cm}^{2}$ \\
\hline
\end{tabular}

a Typical value, depending on excitation

${ }^{\mathrm{b}}$ See Physics World 9, 10 (1996)

' Proposed by C.P.J. Barty at Euroconference, Generation and Application of Ultrashort X-Ray Pulses II, Pisa (1995) fluence, large active volumes are required to generate high-output energies.

In applications related to high-intensity laser-matter interactions, both lasers have their specific advantages. The high photon energy of the $\mathrm{KrF}$ laser radiation favours multiphoton interactions and allows better focusing. The $\mathrm{Ti}$ :sapphire laser system can provide shorter pulses and much higher ponderomotive potentials, which are of interest in a variety of applications. Both laser systems have been successfully applied for the generation of short-pulse VUV and XUV radiation by nonlinear optical processes [6] and as drivers for soft-X-ray lasers [7-9].

In this paper, we discuss applications of a $400 \mathrm{fs}, 20 \mathrm{~mJ}$ $\mathrm{KrF}$ excimer-laser system for the generation of highpower tunable VUV and XUV radiation. In Sect. 1 the $\mathrm{KrF}$ laser system is briefly described. In Sect. 2 recent experimental results on near-resonant four-wave difference-frequency mixing and third-order harmonic generation are presented. Future prospects and speculations about possibilities to use $\mathrm{KrF}$ lasers as drivers for soft-Xray lasers are discussed in Sect. 3.

\section{The short-pulse KrF excimer-laser system}

As has already been mentioned above, femtosecond pulses cannot be generated directly with $\mathrm{KrF}$. They have to be supplied by other longer wavelength laser systems in combination with suitable frequency up-conversion units. For a reliable operation of the $\mathrm{KrF}$ laser system, the shortpulse laser oscillator and the frequency-conversion unit should fulfil some requirements. They should be compact, be able to provide a sufficient amount of energy (about $20 \mu \mathrm{J}$ at $248 \mathrm{~nm}$ ), and their synchronization with the $\mathrm{KrF}$ amplifier should be simple. Among the investigated schemes, the most elegant solution (which has been later used in commercial $\mathrm{KrF}$ laser systems) has been suggested and realized by Schäfer and his coworkers [10]. Their scheme is based on excimer-laser-pumped dye lasers and is integrated into existing commercial double-discharge excimer lasers.

The actual setup of the KrF laser system used in our experiments is shown in Fig. 1. One discharge channel of the excimer-laser (Lambda Physik EMG150ES) is operated as an oscillator at the $\mathrm{XeCl}$ wavelength $(308 \mathrm{~nm})$. This oscillator pumps a sequence of dye lasers, where short pulses (of about $360 \mathrm{fs}$ at a wavelength of $496 \mathrm{~nm}$ ) are generated by special techniques [10] and amplified. These pulses are then frequency-doubled to provide seed pulses at $248 \mathrm{~nm}$ for amplification in the second discharge channel of the excimer laser operating with $\mathrm{KrF}$ as an amplifier. The seed pulses and the $\mathrm{KrF}$ amplifier are synchronized automatically due to the simultaneous operation of the discharges. In commercial systems, typically two-pass on-axis amplification is used, which provides laser pulses with an energy of about $10 \mathrm{~mJ}$. According to proposals and investigations described in [11], our system has been modified to a three-pass off-axis amplification geometry as shown in Fig. 1. This geometry allows to increase the pulse energy up to $20 \mathrm{~mJ}$ with a low ASE background. Parameters of our KrF laser system are summarized in Table 2.

In addition to the $248 \mathrm{~nm}$ pulses, time synchronized $100 \mu \mathrm{J}$ laser pulses at $496 \mathrm{~nm}$ are available. These pulses are used in the near-resonant four-wave frequency mixing described in Sect. 2. Pulses provided by the excimer-laser system are not Fourier-limited, mainly due to the selfphase modulation in the final dye amplifier. It has been shown that the excimer pulses can be compressed down to less than $100 \mathrm{fs}$ [10]. With optimized focusing optics, intensities above $10^{18} \mathrm{~W} / \mathrm{cm}^{2}$ have been demonstrated [11]. This makes KrF laser systems attractive for a wide range of applications. In the experiments on nonlinear optics described below, intensities up to the order of $10^{15} \mathrm{~W} / \mathrm{cm}^{2}$ are used.

\section{Generation of high-power tunable VUV and XUV radiation}

For the generation of high-power tunable VUV and XUV radiation only nonlinear processes can be used at present, since broadly tunable laser systems in this spectral range are not available. With the existing laser systems, two different approaches can be followed: low-order frequency up-conversion (excimer-lasers) or high-order harmonic 


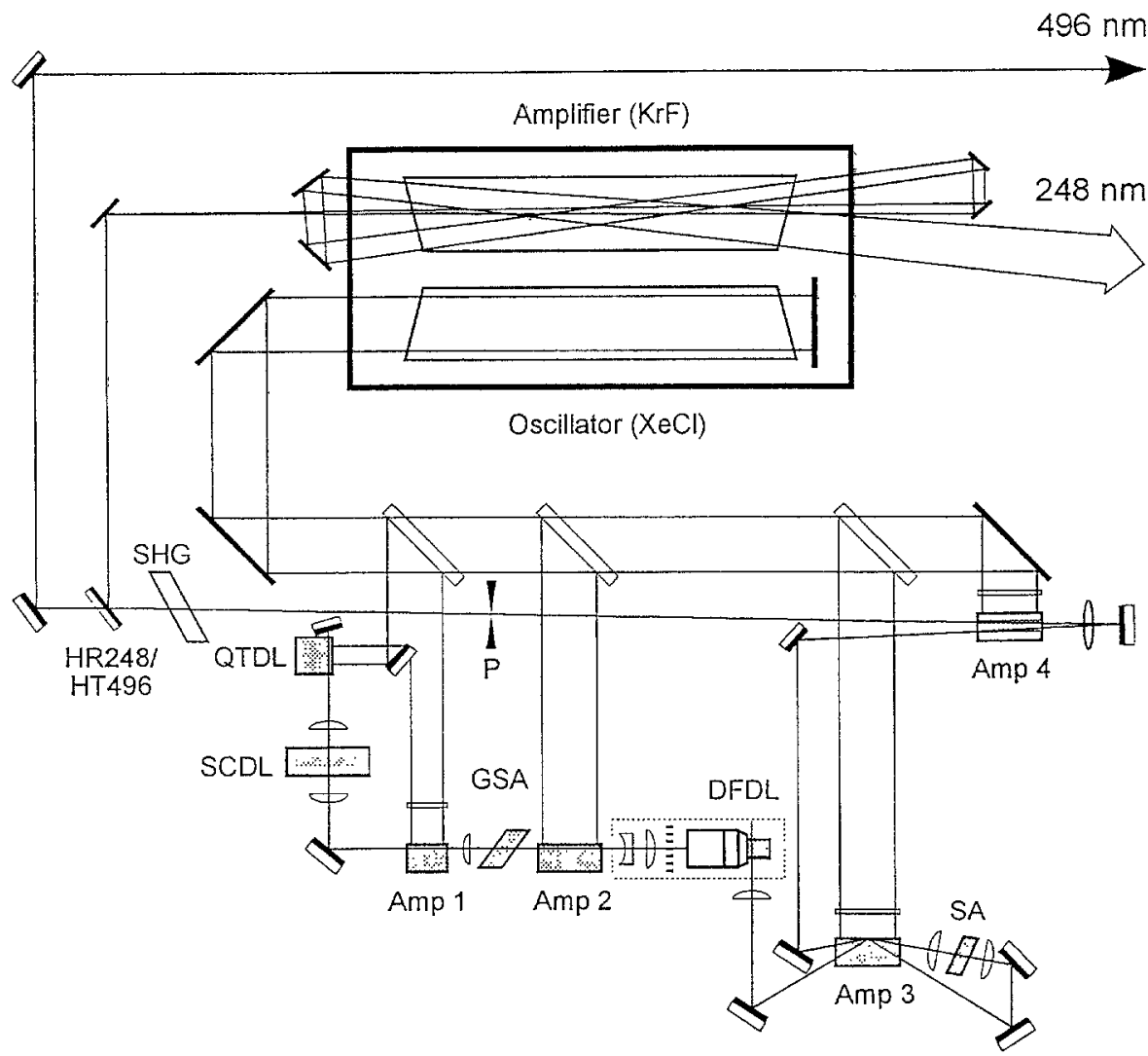

Fig. 1. KrF laser system. Notations: P-pinhole; Amp 1-4 - amplifiers; GSA, Sa - saturable absorbers; SHG - frequencydoubling crystal; QTDL - quenched transient dye laser; SCDL short - cavity dye laser; DFDL - distributed feedback dye laser
Table 2. Parameters of the KrF laser system

\begin{tabular}{lll}
\hline Wavelength & $248 \mathrm{~nm}$ & $496 \mathrm{~nm}$ \\
\hline Pulse energy & $20 \mathrm{~mJ}$ & $0.1 \mathrm{~mJ}$ \\
Pulse duration & $400 \mathrm{fs}$ & $360 \mathrm{fs}$ \\
Maximum peak power & $50 \mathrm{GW}$ & $0.3 \mathrm{GW}$ \\
$\Delta y \Delta t$ & 1.17 & 0.66 \\
Focusability & $M^{2}=3.75$ & $M^{2} \simeq 1$ \\
Beam dimensions & $30 \times 30 \mathrm{~mm}^{2}$ & $3 \mathrm{~mm}$ \\
Polarization & $\mathrm{p}$ & $\mathrm{s}$ \\
Repetition rate & $1-10 \mathrm{~Hz}$ & $1-10 \mathrm{~Hz}$ \\
\hline
\end{tabular}

generation (solid-state lasers). At high laser intensities $\left(>10^{13} \mathrm{~W} / \mathrm{cm}^{2}\right)$, the typical harmonic spectrum consists of an intial steep perturbative decrease, followed by a "plateau" (where the harmonic intensity is almost constant) and a cutoff region (where the harmonic intensity decreases again). For details see [6]. Due to the $\lambda^{2}$-scaling of the harmonic cutoff, a straightforward generation of $\mathrm{X}$-ray radiation (very high-order harmonics) is possible with laser systems operating at longer fundamental wavelengths. However, for the efficient conversion into VUV and XUV radiation, it is preferable to use laser systems operating at shorter wavelengths [12].

The shortest wavelength commercial laser systems are $\mathrm{KrF}(248 \mathrm{~nm}), \operatorname{ArF}(193 \mathrm{~nm})$, and $\mathrm{F}_{2}(157 \mathrm{~nm})$. Studies of short-pulse amplification characteristics for these lasers have shown that only $\mathrm{KrF}$ and $\mathrm{ArF}$ are suited for highpower laser operation [11]. However, in case of ArF the generation of short seed pulses is more complicated [13], and compact commercial systems are not available. At present, the above-described $\mathrm{KrF}$ laser system seems to be the most convenient tool for the efficient generation of powerful VUV and XUV radiation by low-order nonlinear processes.

In the perturbative regime, the nonlinear polarization $\mathscr{P}(t)$ of a gaseous medium in the laser field $\mathscr{E}(t)$ can be written as

$\mathscr{P}(t)=\chi^{(1)} \mathscr{E}(t)+\chi^{(3)} \mathscr{E}^{3}(t)+\chi^{(5)} \mathscr{E}^{5}(t)+\cdots$,

where $\chi^{(1)}$ is the linear susceptibility (a second-rank tensor), and $\chi^{(3)}$ and $\chi^{(5)}$ are the third- and fifth-order nonlinear optical susceptibilities (a fourth-rank tensor, etc.). High-order terms in the nonlinear polarization lead to the generation of corresponding order harmonics. To enhance the intensity of a specific harmonic order, phase matching between the driving polarization and the generated harmonic field is necessary. Using resonances of the corresponding nonlinear susceptibilities, the conversion efficiencies can be further increased.

\subsection{Near-resonant four-wave difference-frequency mixing}

From nonlinear optical experiments with ns-laser pulses it is well known that the use of two-photon resonances is advantageous in third-order nonlinear processes [14], while the one- and three-photon resonances are associated with strong linear absorption of the incident and the generated field, respectively. For shorter pulses and higher intensities, it is preferable to avoid exact resonances, because they can lead to different competing processes 

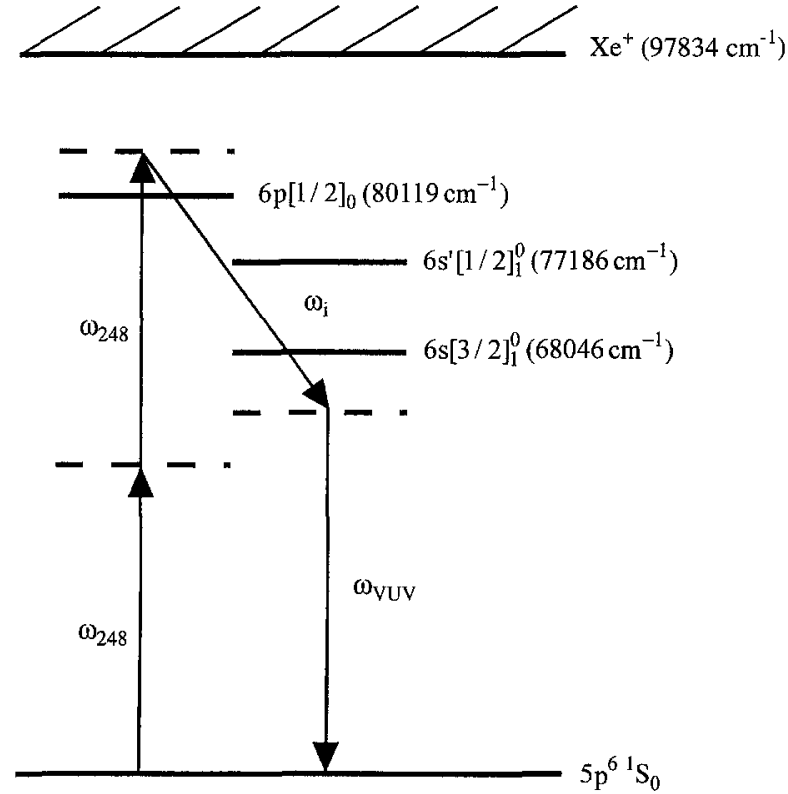

Fig. 2. Resonantly enhanced four-wave mixing scheme in Xe used for the generation of VUV femtosecond pulses $\left(\omega_{\mathrm{VUV}}=2 \omega_{248}-\omega_{\mathrm{i}}\right)$

(depletion of the ground state by two-photon absorption and two-step ionization, self-starting parametric and Raman processes, etc.). At high intensities, a near twophoton resonance therefore seems to be a good compromise. As can be seen in Fig. 2, for $\mathrm{KrF}$ laser radiation such a near two-photon resonance exists in $\mathrm{Xe}$ for the $5 p^{6}-5 p^{5} 6 p\left[\frac{1}{2}\right]_{0}$ transition. By excitation of Xe near this resonance, a strong short-living nonlinear polarization can be produced. This polarization may drive a self-starting four-wave parametric process, which allows to generate short-pulse broadband VUV radiation around $148.5 \mathrm{~nm}$ (signal) and radiation around $760 \mathrm{~nm}$ (idler), as has been demonstrated in [15]. In order to generate tunable VUV radiation by Four-Wave Difference-Frequency Mixing (FWDM) according to $\omega_{\mathrm{vuv}}=2 \omega_{248}-\omega_{\mathrm{i}}$, an additional tunable laser field $\omega_{\mathrm{i}}$ (idler field) is necessary (Fig. 2). Short-pulse fixed frequency and tunable VUV radiation in the spectral range of about $200-133 \mathrm{~nm}$ (with $\omega_{\mathrm{i}}$ ranging from $330 \mathrm{~nm}$ up to about $1.9 \mu \mathrm{m}$ ) has been demonstrated in a variety of experiments [16-18]. Nanosecond and subpicosecond pulses have been applied as an idler. In these experiments, Xe gas cells with pressures around 50 mbar have been used. FWDM has been performed in a non-collinear-phase matching geometry (with matching angles below $1^{\circ}$ ) [16]. Due to the small angles, the VUV radiation is almost collinear with the $\mathrm{KrF}$ laser radiation. With pump energies of about $10 \mathrm{~mJ}$ at $248 \mathrm{~nm}$ focused to intensities of $10^{15} \mathrm{~W} / \mathrm{cm}^{2}$ and $\mu J$ energies of subpicosecond idler pulses, VUV radiation with energies of about $1 \mu \mathrm{J}$ can be easily produced. For example, with tunable idler radiation of $16 \mu \mathrm{J}$ at $590 \mathrm{~nm}$, tunable subpicosecond pulses with energies up to $7 \mu \mathrm{J}$ at $157 \mathrm{~nm}$ have been obtained (Fig. 3). Short-pulse VUV radiation generated by FWDM in Xe has been used to study the short-pulse amplification behaviour of ArF $(193 \mathrm{~nm})$ and $F_{2}(157 \mathrm{~nm})$ amplifiers $[19,20]$.

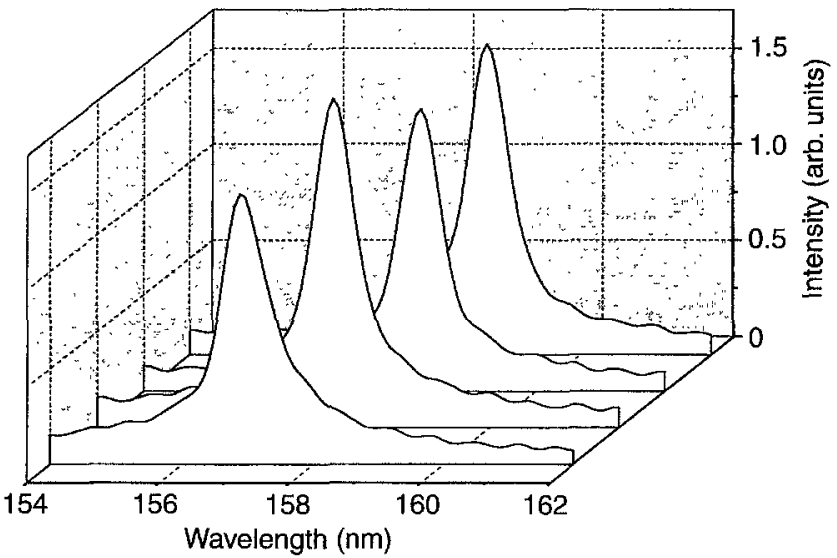

Fig. 3. Spectra of VUV radiation generated by FWDM in Xe tuned around $157 \mathrm{~nm}$

From calculations and experimental results on FWDM, the nonlinear susceptibilities can be obtained. For example, at $155 \mathrm{~nm}$ the nonlinear susceptibility is given by $\chi^{(3)}$ $\left(-\omega_{155} ; \omega_{248}, \omega_{248},-\omega_{626}\right)=-6 \times 10^{-49} \mathrm{~m}^{5} / \mathrm{v}^{2}$. Taking this value and pump intensities of the order of $10^{13} \mathrm{~W} / \mathrm{cm}^{2}$, conversion efficiencies of the order of several percents (with respect to the $\mathrm{KrF}$ radiation) and output powers at the GW level can be estimated. In order to achieve these values, the energy (power) of the tunable injection field (idler) has to be increased, the phase-matching geometry has to be optimized, and various losses due to competing processes should be reduced.

In our present experiments, for the generation of highpower idler radiation an Optical Parametric Generator (OPG) pumped with $100 \mu \mathrm{J}$ radiation at $496 \mathrm{~nm}$ from the $\mathrm{KrF}$ laser system, followed by a two-stage dye amplifier, is used. The experimental setup is shown in Fig. 4. For the OPG, a BBO crystal with type-II phase matching and a double-pass geometry has been chosen. The pumping with $496 \mathrm{~nm}$ radiation guarantees an exact synchronization of the OPG and KrF laser pulses. With the OPG, pulse energies of about $3 \mu \mathrm{J}$, tunable in the range of $600 \mathrm{~nm}$ to $>1 \mu \mathrm{m}$ (signal and idler) are produced. With the two-step dye amplifier (laser dye:DCM), pumped by about $150 \mathrm{~mJ}$ of a frequency-doubled $\mathrm{Nd}: Y A G$ laser $(8 \mathrm{~ns})$, amplification up to about $400 \mu \mathrm{J}$ is achieved. A complete tuning curve of the high-power OPG-dye system is shown in Fig. 5. The output pulses typically have a bandwidth of $3 \mathrm{~nm}$ and a pulse duration of about $750 \mathrm{fs}$. These pulses are not Fourier-limited, due to the initial chirp of the $496 \mathrm{~nm}$ pulses and further self-phase modulation in the dye amplifier. By compression with a prism compressor, a pulse duration of $250 \mathrm{fs}$ has been measured. Thus, idler radiation with a peak power of more than one GW can be produced with the OPG-dye system.

With the tuning range of the idler radiation shown in Fig. 5, a VUV range from 151.8 to $156 \mathrm{~nm}$ can be covered by the difference-frequency mixing process. To achieve high-output energies in the VUV, optimization experiments have been performed with idler pulses at $626 \mathrm{~nm}$, leading to VUV radiation at $155 \mathrm{~nm}$ [21]. An important step in the optimization of the produced VUV radiation is 


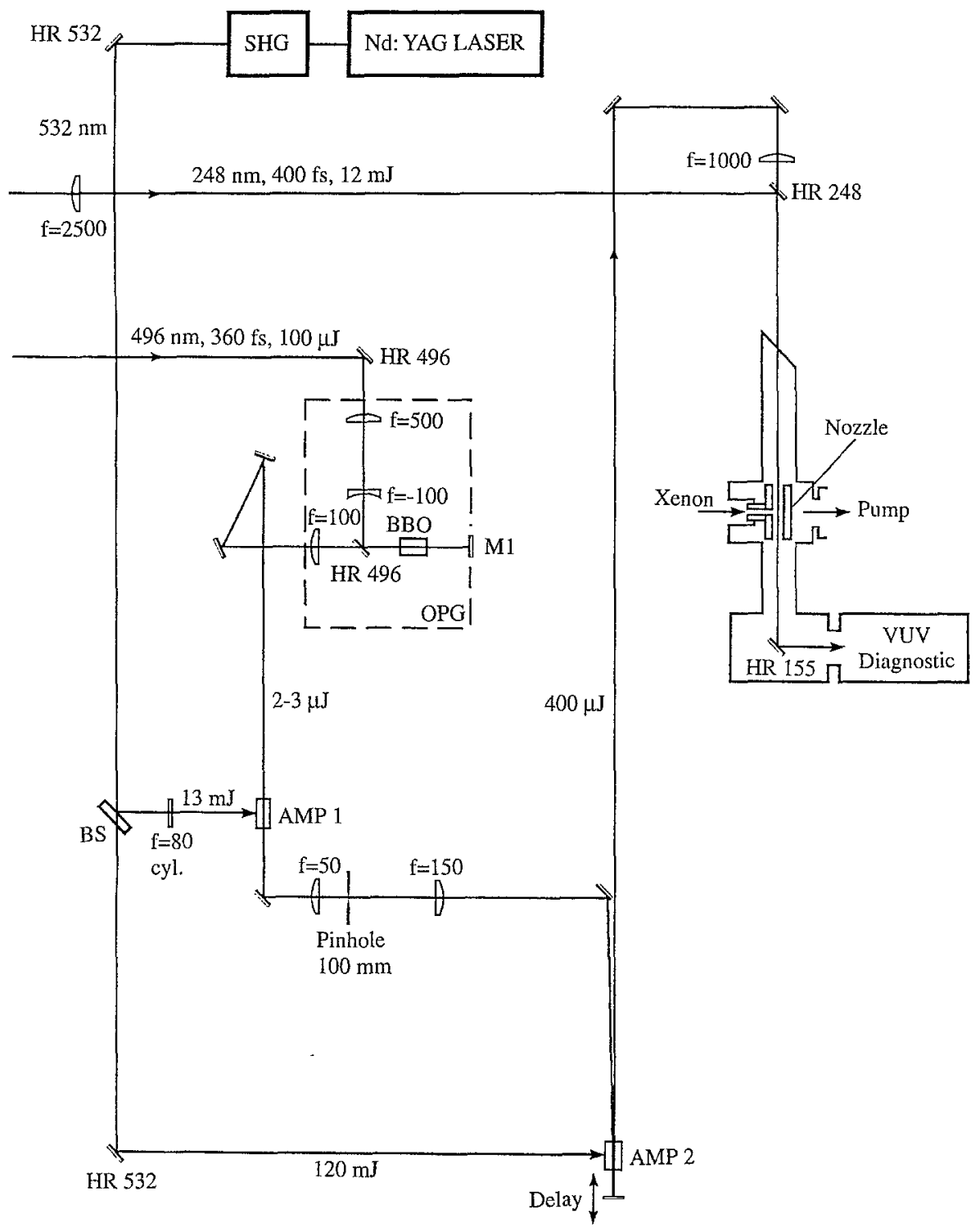

Fig. 4. Experimental setup for the generation of tunable short-pulse VUV radiation. Amp 1-2 - dye amplifiers; M1 - dielectric broadband mirror

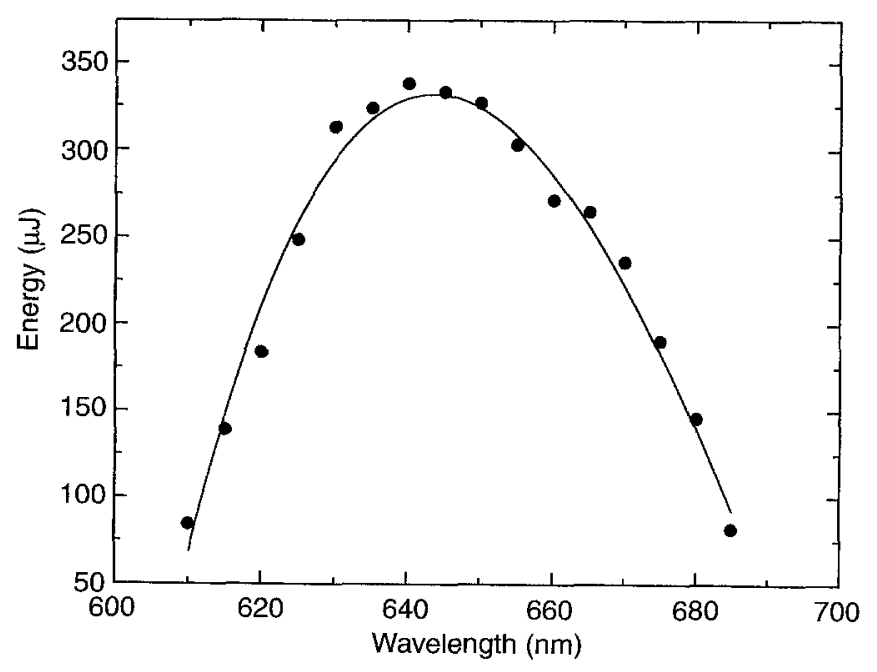

Fig. 5. Tuning curve of amplified OPG pulses (laser dye: DCM) the use of a pulsed Xe gas jet instead of a cell, which allows to increase the output energy by a factor of 2 . The design of the gas jet and the whole VUV generation scheme is shown in more detail in Fig. 6. The gas jet is formed by an open cylindrical tube into which the Xe gas is injected from a pulsed commercial valve (General Valve Corporation). The valve is triggered approximately $700 \mu$ s before the laser pulses enter the cylindrical tube. An optimum VUV output energy is obtained for a backing pressure of about 5 bar, which corresponds to a Xe pressure in the tube of about 40 mbar. This is in agreement with the typical pressures used in Xe cells. For the chosen noncollinear mixing geometry and focusing optics $\left(f_{248}=\right.$ $2 \mathrm{~m}, f_{626}=1 \mathrm{~m}$, phase-matching angle $0.7^{\circ}$ ), a tube diameter of $3 \mathrm{~mm}$ and a length of $5 \mathrm{~cm}$ have been used. This tube length corresponds to the interaction length of the laser beams in the non-collinear geometry. Outside the gas tube the Xe pressure rapidly decreases. This allows to reduce losses of the pump and generated VUV radiation via self-absorption in Xe gas. In addition, no exit window is required for the gas jet, which avoids losses in the 


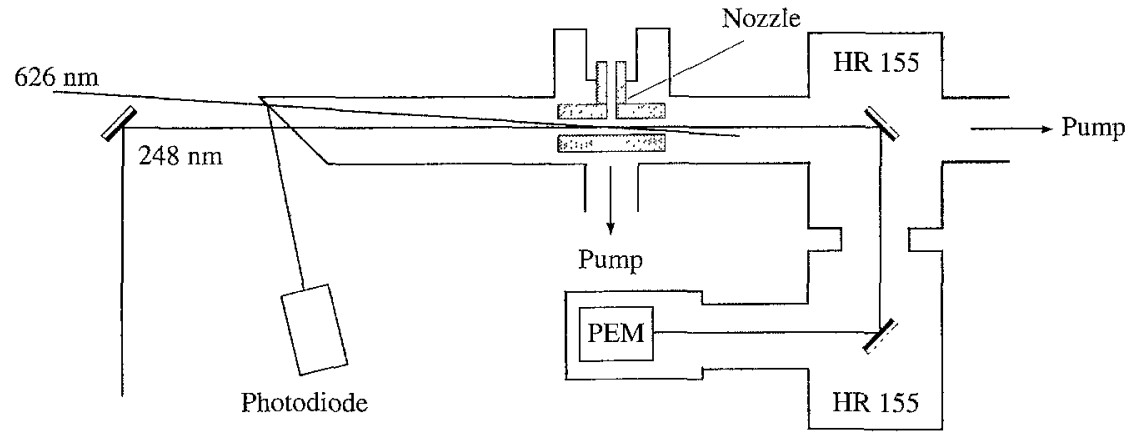

Fig. 6. Noncollinear mixing geometry and nozzle design for the mixing process: PEMenergy detector

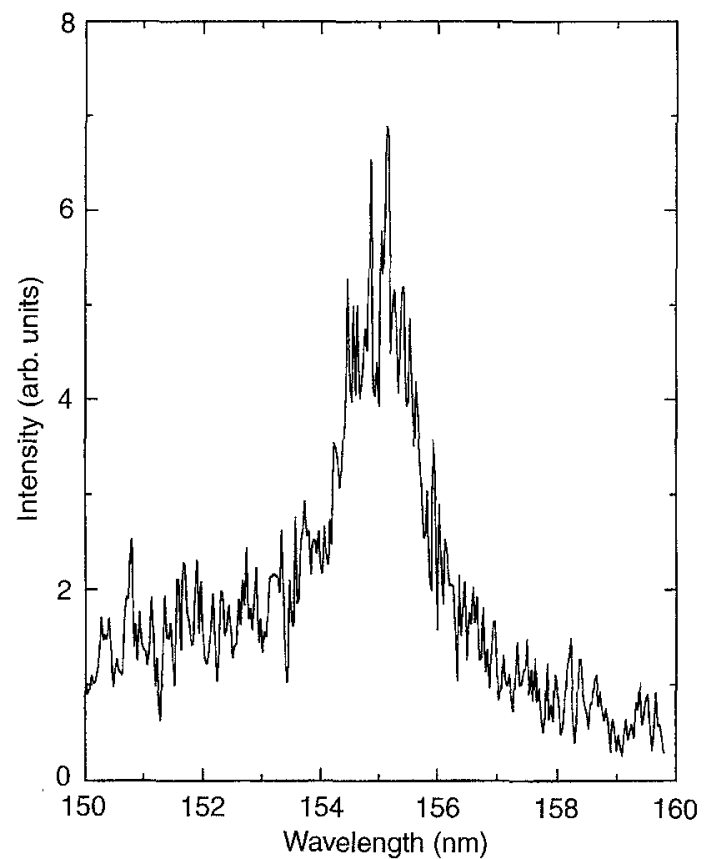

Fig. 7. Multishot spectrum of FWDM radiation at $155 \mathrm{~nm}$

window material $\left(\mathrm{MgF}_{2}\right)$. To achieve maximum VUV energies, the pump pulses should overlap in time and space. With a dichroic mirror the generated VUV radiation is separated from the pump radiation and is detected by a VUV monochromator.

In Fig. 7, a multishot VUV spectrum at $155 \mathrm{~nm}$ with a bandwidth of $1 \mathrm{~nm}$ is shown. Although the time duration of the generated VUV pulses could not be measured so far, experimental results from $[16,18]$ suggest a pulse duration $\leq 400$ fs for the generated VUV radiation. According to the duration of pump pulses, a minimum VUV pulse duration of $230 \mathrm{fs}$ is expected. Of special interest was the energy of the VUV radiation. In Fig. 8, the dependence of the VUV energy on the energy of the idler pulses is shown, at fixed parameters of the $\mathrm{KrF}$ laser radiation (energy, focusing geometry, pulse duration, etc.). An almost linear dependence with no saturation is observed, in agreement with a simple perturbative description of the FWDM process. At the idler energy of $\sim 300 \mu \mathrm{J}$ and a KrF pulse energy of 10-15 mJ, a VUV energy of $260 \mu \mathrm{J}$ is measured [21]. Assuming a pulse duration of $230 \mathrm{fs}$, this corresponds to a peak power of $\sim 1 \mathrm{GW}$ and a conversion

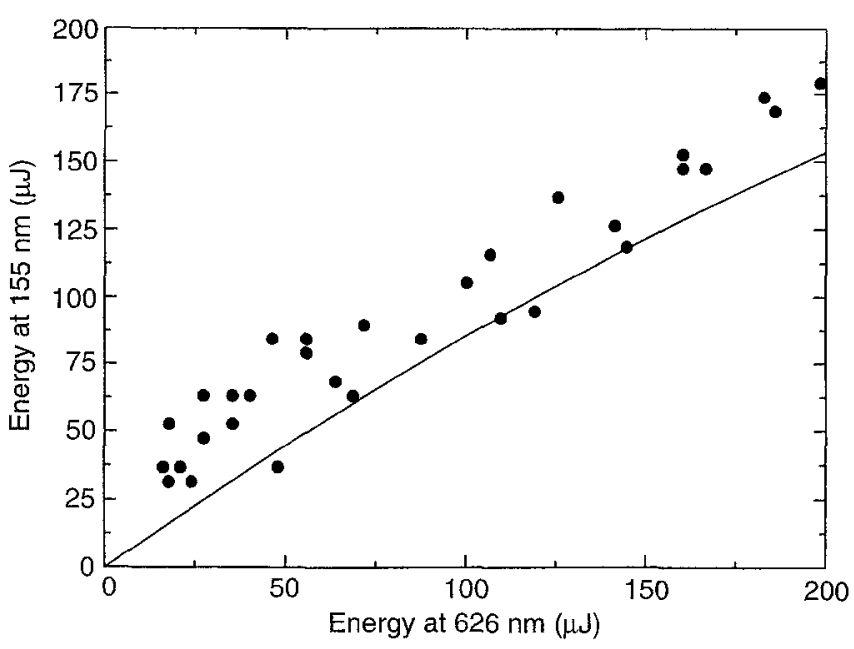

Fig. 8. Output energy at $155 \mathrm{~nm}$ vs input energy at $626 \mathrm{~nm}$. Solid curve shows numerical simulation results (see also Fig. 9)

efficiency (with respect to the $\mathrm{KrF}$ radiation) of $2 \%$, which so far has not been demonstrated for a conversion process into the VUV.

This powerful VUV radiation can be applied in nonlinear spectroscopy, for example, in investigations of ionization and dissociation of molecules. The present tuning range of 151.8-156 nm can easily be extended by using other dyes in the dye amplifier. Note that even without the dye amplifier, the system delivers sufficient VUV radiation (up to $1 \mu \mathrm{J}$ ), which is tunable in the range of $\sim 130-200 \mathrm{~nm}$ (including a frequency doubling of the idler radiation) with a small gap around the $5 p^{6}-5 p^{5} 6 s\left[\frac{3}{2}\right]_{0}$ Xe resonance at $147 \mathrm{~nm}$.

Our special interest concerns the development of this VUV radiation source towards still higher energies and its application for further frequency up-conversion into XUV spectral range. The measured linear dependence of the VUV radiation on the idler energy (Fig. 8) allows to conclude that by increasing the idler energy, a further increase of the VUV energy should be possible. To verify this, numerical simulations of the FWDM process have been performed, which are in good agreement with experimental results. Nonlinear differential equations for the Gaussian pump, idler and generated fields has been solved, taking into account propagation effects (phase matching, influence of the Kerr effect on the pump beam 


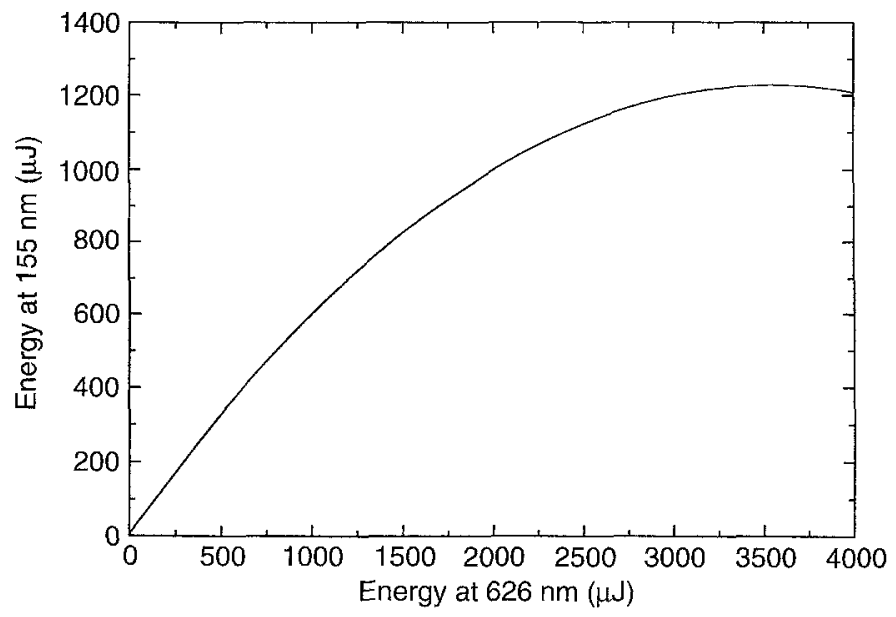

Fig. 9. Further energy scaling of the FWDM process for our experimental conditions.

profile, etc.) and two-photon absorption and ionization processes. For our experimental conditions, these simulations predict (Fig. 9) a saturation of the VUV radiation at an energy level of $1 \mathrm{~mJ}$ for an idler energy of 3-4 mJ. The energy of the $\mathrm{KrF}$ pump radiation reduces during the FWDM process from 10 to $2 \mathrm{~mJ}$. Recall that in the FWDM process also the idler energy is amplified (by about $1 \mathrm{~mJ}$ ). To avoid the saturation of the generated VUV radiation, the pump energy should be further increased, which then also requires a new optimization of the focusing and phase matching conditions. To realize a $1 \mathrm{~mJ}$ VUV source, a novel amplifier scheme for the idler radiation is presently developed. In this scheme a multipass Ti:sapphire amplifier will be used, which should produce broadly tunable idler radiation from 670 to $1070 \mathrm{~nm}$ corresponding to a VUV frequency range from 140.6 to $152.5 \mathrm{~nm}$.

\subsection{Third-order harmonic generation}

Now we turn to a discussion of the generation of shortpulse coherent XUV radiation. For this low-order nonlinear processes with the $\mathrm{KrF}$ laser radiation or the abovedescribed VUV radiation (or with different combination of both) can be applied.

To evaluate the potential of the low-order nonlinear frequency up-conversion, direct frequency tripling of the $\mathrm{KrF}$ laser radiation has been investigated. The $\mathrm{KrF}$ pulses were focused into similar cylindrical gas jets, as shown in Fig. 6. As nonlinear media the rare gases have been used.

Our results on the third-harmonic generation are presented in Fig. 10. In general, the third-harmonic signal decreases towards the light rare gases and follows the corresponding behaviour of the static polarizabilities given in Table 3. In this row only Ar gas strongly deviates. In Ar the third-harmonic signal is much stronger. This can be explained by a near three-photon resonance enhancement of the corresponding third-oder susceptibility ( Fig. 11). In the lighter rare gases these resonant conditions are not fulfilled. In the heavier rare gases $(\mathrm{Kr}, \mathrm{Xe})$ three-

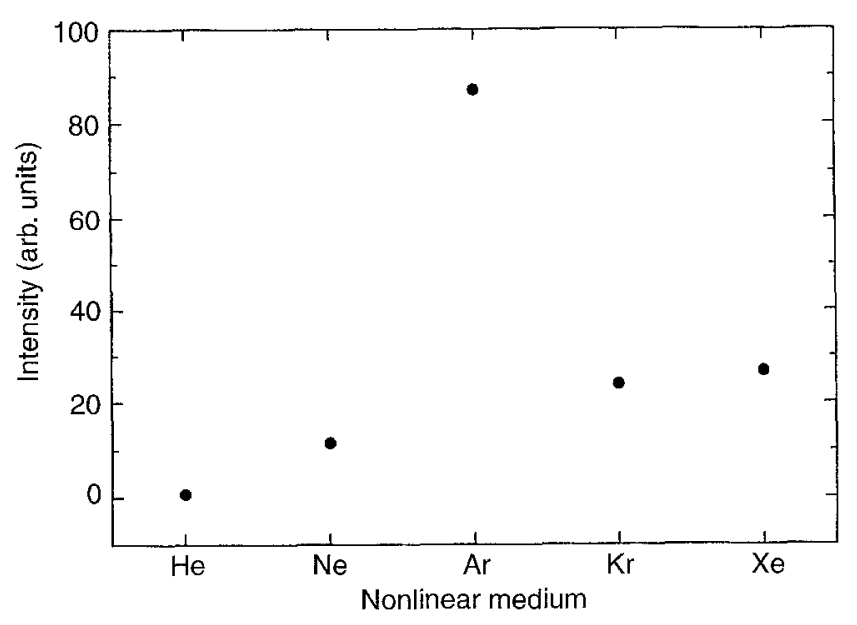

Fig. 10. Variation of the XUV signal at $83 \mathrm{~nm}$ for different rare gases

Table 3. Ionization energies and static polarizabilities for rare gases (from CRC Handbook of Chemistry and Physics)

\begin{tabular}{lccccc}
\hline Gas & $\mathrm{He}$ & $\mathrm{Ne}$ & $\mathrm{Ar}$ & $\mathrm{Kr}$ & $\mathrm{Xe}$ \\
\hline$I_{0}[\mathrm{eV}]$ & 24.58 & 21.56 & 15.76 & 14.00 & 12.13 \\
$\alpha\left[10^{-24} \mathrm{~cm}^{3}\right]$ & 0.205 & 0.396 & 1.641 & 2.484 & 4.044 \\
\hline
\end{tabular}

photon transitions lie already above the ionization threshold. In this case, ionization losses of the pump (threephoton) and of the generated harmonic (single-photon) radiation may reduce the conversion efficiency.

With $8 \mathrm{~mJ} \mathrm{KrF}$ pump laser radiation, focused by a $2 \mathrm{~m}$ lens to an intensity of about $10^{15} \mathrm{~W} / \mathrm{cm}^{2}$ (confocal parameter $2.5 \mathrm{~mm}$ ), a maximum XUV energy of $14 \mu \mathrm{J}$ has been obtained. In this experiment, the cylindrical Ar gas jet (length $5 \mathrm{~cm}$, diameter $2.5 \mathrm{~mm}$ ) and optimized conditions (a backing pressure of 3 bar, atomic density in the gas jet of $10^{17}-10^{18} \mathrm{~cm}^{-3}$ ) have been used. The energy measurements have been performed with a calibrated XUV photodiode. The measured energy of $14 \mu \mathrm{J}$ corresponds to a conversion efficiency of $\sim 2 \times 10^{-3}$ and a peak power of about $40 \mathrm{MW}$ for a pulse duration of $400 \mathrm{fs}$. Note that, in principle, shorter XUV pulses are expected [22]. The measured bandwidth of the third-harmonic signal is $0.4 \mathrm{~nm}$ (Fig. 12), which is above a calculated bandwidth of $0.12 \mathrm{~nm}$ taking into account the initial bandwidth of $0.6 \mathrm{~nm}$ of the $\mathrm{KrF}$ system. This broadening can be partially explained by a spectral blue shift in an ionizing medium [18]. An analysis of the phase-matching conditions in Ar indicates that a slight frequency shift of the maximum of the $\mathrm{KrF}$ laser radiation spectrum, within its gain profile, should result in an improved phase matching and a further increase in the efficiency of the frequencytripling process.

The results presented above demonstrate the high potential of low-order nonlinear processes for the generation of XUV radiation. This allows one to hope that other conversion schemes (see below) will also be effective. It is clear that to obtain tunable XUV radiation, frequency 


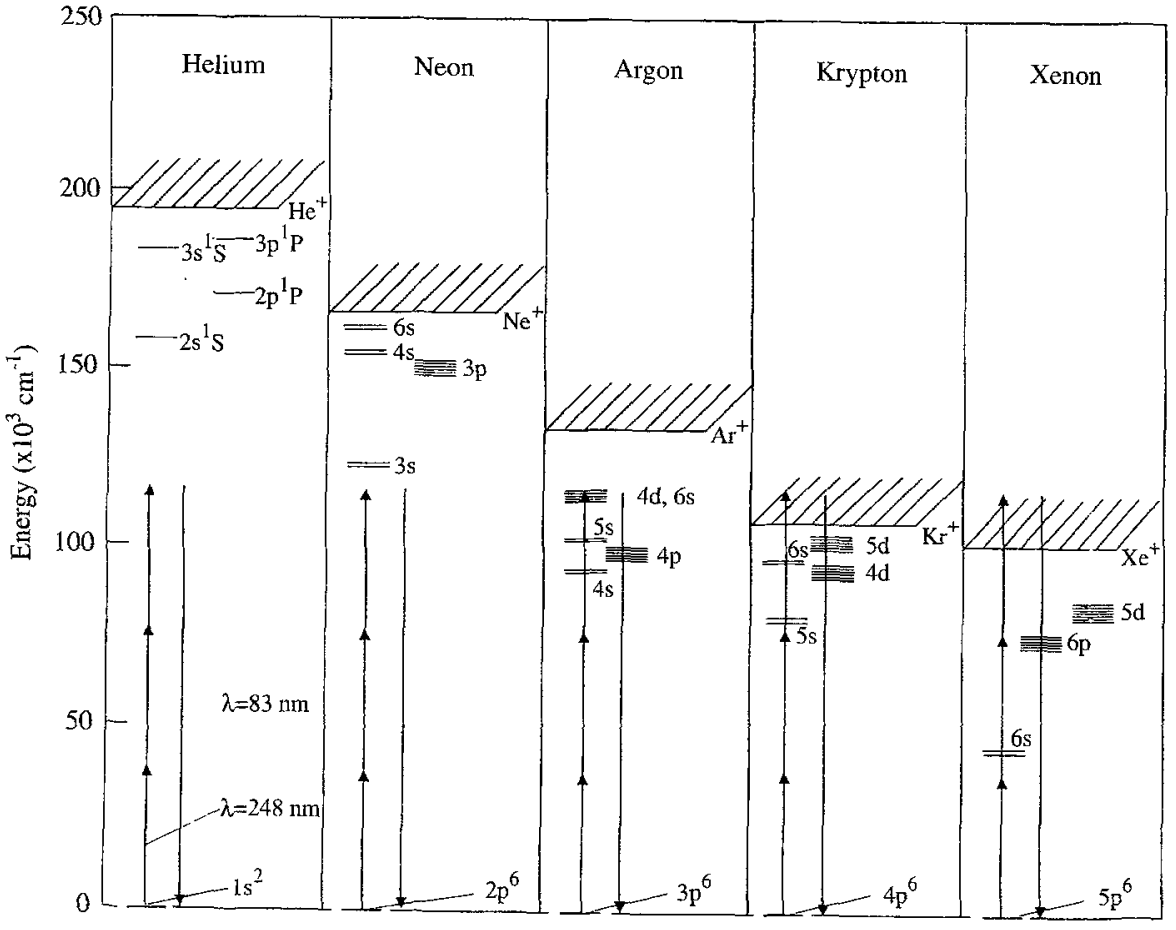

Fig. 11. Reduced energy level diagrams for rare gases

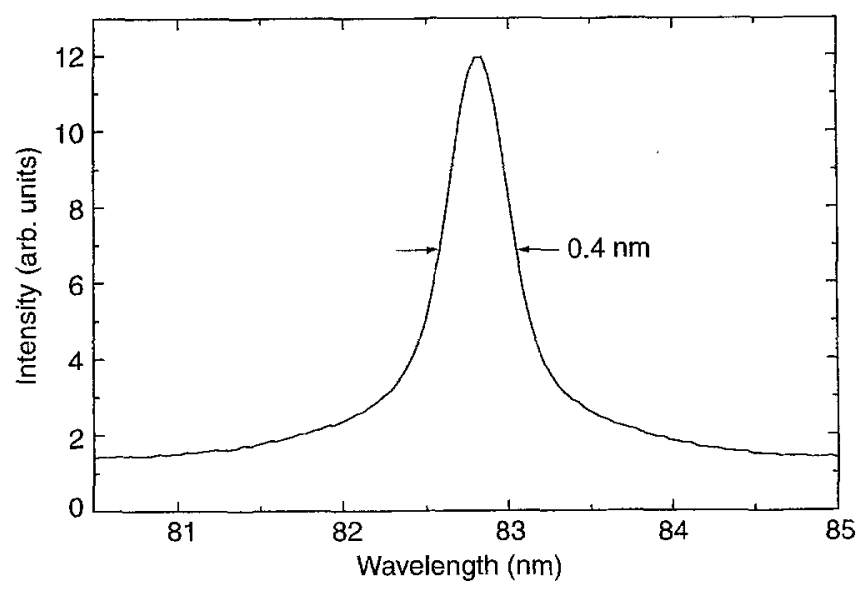

Fig. 12. Single-shot XUV spectrum of frequency tripled femtosecond radiation at $248 \mathrm{~nm}$

up-conversion should be performed with already tunable radiation fields. In Fig. 13 we summarize nonlinear processes which are presently investigated and their potential tuning ranges. For the realization of these processes, nonlinear materials with near-resonant conditions are required. For the fifth-order process, $\omega_{\mathrm{xuv}}=4 \omega_{248} \pm \omega_{\mathrm{i}}$, the two-photon resonance in $\mathrm{Xe}$ and the tree-photon resonance in Ar can be used. In first preliminary experiments, strong signals at $56.6 \mathrm{~nm}$ for the fifth-order sumfrequency mixing and at $68.8 \mathrm{~nm}$ for the difference-frequency mixing have been observed in Xe. More detailed investigations on this subject are planned in the near future. With the low-order frequency mixing processes we expect to obtain output energies of $0.1-1 \mu \mathrm{J}$ in the XUV spectral range.

Closing this section we note that a low-charged plasma can become an interesting nonlinear medium for low-order frequency mixing. The nonlinear polarizability of low-charged ions is still high enough. Moreover, in this case new resonant transitions can be found. The fact that the plasma has a definite positive dispersion (due to free electrons) can be considered as an additional advantage. It is well known that in a medium with positive dispersion perfect phase matching can be realized for difference-frequency mixing processes. Recent experimental results $[23,24]$ allow to be very optimistic on this subject. 


\section{Prospects for soft-X-ray lasers pumped by short-pulse $\mathrm{KrF}$ laser radiation}

Merits of short-pulse $\mathrm{KrF}$ laser systems for the realization of soft-X-ray lasers have been early realized by Schäfer and his Laser Physics Group at the Max-Planck-Institut für biophysikalische Chemie [25]. For this goal they have started and performed important developments of laboratory-scale powerful $\mathrm{KrF}$ laser systems of the type, which has been described above. In 1993, this development has allowed another group to demonstrate, for the first time, amplified spontaneous emission on the Lyman- $\alpha$ transition $(13.5 \mathrm{~nm})$ in $\mathrm{H}$-like $\mathrm{Li}$ ions [7]. In this experiment, $0.5 \mathrm{ps}, 50 \mathrm{~mJ} \mathrm{KrF}$ laser radiation was focused into a singly ionized lithium plasma at an intensity of $10^{17}$ $\mathrm{W} / \mathrm{cm}^{2}$. In this section, we briefly describe this and other possibilities to use short-pulse KrF systems as drivers for soft-X-ray lasers.

Present short-pulse driven soft-X-ray lasers are based on tunnel ionization of atoms in an intense laser field. This ionization mechanism allows to strip all atoms to a necessary, definite ionization stage and even to prepare a desired electron energy distribution. The last can be done simply by varying the polarization of the laser field. In the case of a linearly polarized laser field, the ionized free electrons can be left relatively cold, which allows to create a transient population inversion between excited and ground-ion states during the three-body recombination cascade. This approach has been used in [7] and later in [9]. In the case of a circularly polarized laser field, the ionized electrons are hot and can provide effective collisional excitation pumping. This has been recently demonstrated in [8].

In all experiments, for the realization of the abovediscussed tunnel ionization schemes, a confocal geometry (with a longitudinal pumping of an active medium) has been used. In this geometry it is advantageous to use UV $\mathrm{KrF}$ laser pulses $(\omega=5 \mathrm{eV})$ due to their better focusability. Moreover, due to the very high critical plasma density, $N_{\mathrm{e}}=1.8 \times 10^{22} \mathrm{~cm}^{-3}$, for the $\mathrm{KrF}$ laser radiation, defocusing and other self-action effects are minimized.

For ionization to occur in the tunnelling regime, the condition $\gamma=\left(E_{\mathrm{i}} / 2 U_{\mathrm{p}}\right)^{1 / 2}<1$ should be fulfilled, where $\gamma$ is the Keldysh parameter [26], and $E_{\mathrm{i}}$ and $U_{\mathrm{p}}$ are the ionization and ponderomotive potentials. Using expressions for the ion appearance intensity given by the overbarrier model [27], the above condition can be written as $E_{\mathrm{i}}>4 R y^{1 / 3}(Z \omega)^{2 / 3}$, where $\omega, E_{\mathrm{i}}$, and $R y=13.6$ are in $\mathrm{eV}$, and $Z$ is the charge of the produced ion. In this case, the Keldysh parameter is given by $\gamma=8 Z \omega\left(R y / E_{\mathrm{i}}^{3}\right)^{1 / 2}$. The electron temperature after tunnel ionization in a linearly polarized laser field can be estimated from (see [9] for details)

$T_{\mathrm{e}}=2^{-10} \frac{E_{\mathrm{i}}^{9 / 2}}{Z^{3} \omega^{2} R y^{3 / 2}}[\mathrm{eV}]$.

For $\mathrm{KrF}$ lasers, the applicability of the tunnel ionization approach requires $E_{\mathrm{j}}>28 \times Z^{2 / 3}[\mathrm{eV}]$, which can be fulfilled only for multiply charged ions. Ionization of atoms (and molecules) in $\mathrm{KrF}$ laser fields occurs in the multiphoton regime $(\gamma>1)$. Therefore, for the realization of soft-X-ray lasers based on tunnel ionization, a lowtemperature plasma should be used as a target in case of $\mathrm{KrF}$ laser pulses. In [7] a singly ionized Li plasma produced by a $20 \mathrm{~ns} \mathrm{KrF}$ laser pulse has been used for this goal.

For gaseous targets it seems to be more advantageous to use a hybrid $\mathrm{KrF} / \mathrm{Ti}$ : sapphire laser system, where frequency-tripled Ti:sapphire laser pulses are used to seed a KrF amplifier. Such a system has been first demonstrated in 1993 [28] and allows to combine advantages of $\mathrm{Ti}$ :sapphire and $\mathrm{KrF}$ lasers. Hybrid geometry provides short pulses with low ASE background and allows synchronization of $\mathrm{Ti}$ :sapphire and $\mathrm{KrF}$ laser pulses. Potential applications of this system for the realization of soft-X-ray lasers are very attractive. The following two-pulse scenario can be used for this goal. First, linearly polarized Ti:sapphire laser radiation (fundamental, $\omega \simeq 1.67 \mathrm{eV}$ ) is focused into a gas jet (or cell) to produce a low-temperature plasma (preplasma). In this case, optical field ionization occurs in the tunnelling regime and the electron temperature is given by (2). Then, more powerful $\mathrm{KrF}$ laser radiation is used to ionize the preplasma to a higher ionization stage. As an example, we discuss here plasma parameters, which can be produced by these two pulses in Xe gas. In Fig. 14a the variation of the ionization potential with the ion charge is shown for $\mathrm{Xe}$. The ionization potential varies approximately

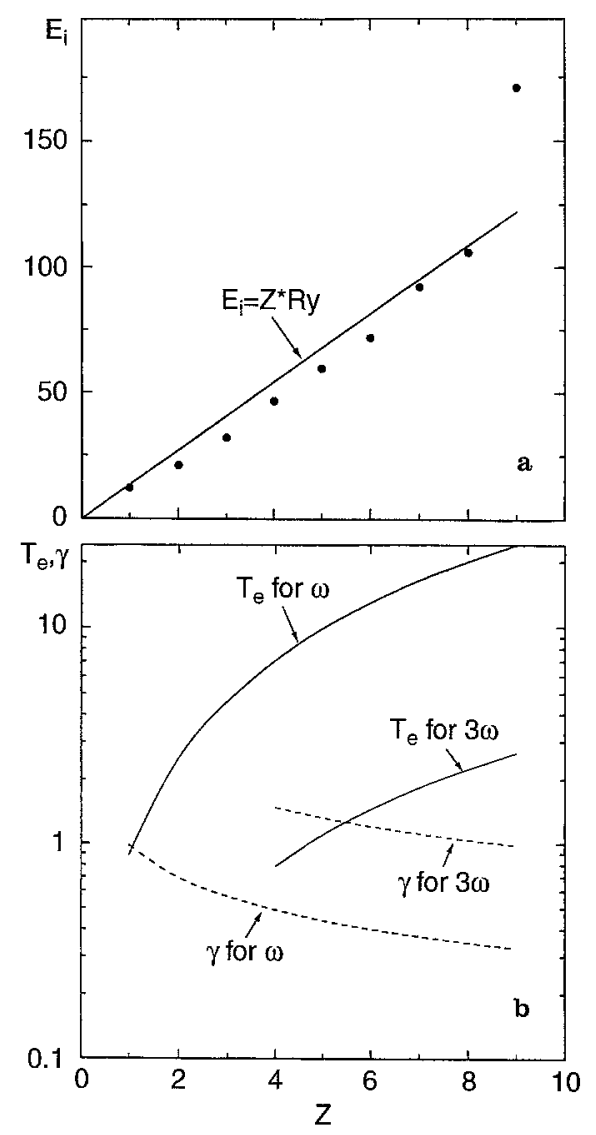

Fig. 14a. Ionization potentials for $\mathrm{Xe}$ ions. Solid line shows $E_{\mathrm{i}}=Z \times R y$. (b) Electron temperatures and Keldysh parameters for $\mathrm{Xe}$ ions 
as $E_{\mathrm{i}}=Z \times R y$, which allows a simple estimate of the electron temperature as $T_{\mathrm{e}} \simeq 2^{-10} R y^{3} Z^{3 / 2} / \omega^{2}$ [eV]. In Fig. 14b electron temperatures and Keldysh parameters $\gamma$ are shown for different Xe ions. As can be seen, when other heating mechanisms can be neglected, the two-pulse scheme allows to keep the electron temperature below $5 \mathrm{eV}$. Thus, using a two-pulse scheme more cold plasmas can be created with linearly polarized radiation and a better "adjustment" of the electron temperature is possible with two circularly polarized pulses.

In case of $\mathrm{KrF}$ laser radiation $(3 \omega)$, the Keldysh parameter $\gamma$, shown in Fig. 14b, is of the order of 1 even for relatively high ion states, $Z=5-9$. This means that the ionization is in a transient (multiphoton-tunnel) ionization regime. For $\gamma \sim 1$ (and even smaller) multiphoton processes can play an important role, since the strict applicability of tunnel ionization model requires $\gamma \ll 1$.

In the multiphoton regime of laser-atom (laser-ion) interactions, which is very often realized for $\mathrm{KrF}$ lasers, additional mechanisms can lead to the creation of inversion with respect to the ion ground state. Here we consider a resonant multiphoton excitation of upper ion states as a possible pumping mechanism for soft-X-ray lasers.

Several years ago the role of resonance effects in multiphoton and above-threshold ionization of atoms has been a subject of intense discussions $[29,30]$. Experiments on muliphoton ionization in very intense laser fields have revealed resonance structures, when ac Stark shifts of the excited levels are comparable or larger than the photon energy. These structures appear due to the intensitydependent ac Stark effect which shifts different atomic levels into a multiphoton resonance. It has even been declared in [29] that all high-intensity multiphoton ionization is resonant. In [31] large populations in excited states after short-pulse multiphoton ionization have been observed.

It is well known that in case of incoherent interaction of a two-level atom with a resonant optical field, populations of the ground and excited levels become equal $\left(N_{0} / g_{0}=N_{1} / g_{1}\right.$, where $N=N_{0}+N_{1}$ is the initial number of atoms in the ground state, and $g_{0}$ and $g_{1}$ are the corresponding statistical weights). A multiphoton resonance in a sufficiently strong laser field can also lead to an analogous equilibration of populations. Here we remind, first, how the resonance conditions are modified in the presence of a strong laser field.

In the strong laser field, $\mathscr{E}(t)=\mathscr{E}_{0}(t) \cos \omega t$, with the time dependent field amplitude $\mathscr{E}_{0}(t)$, a single atomic level experiences the ac Stark shift $\delta E_{n}(t)$, which is defined by its polarizability $\alpha_{n}=\chi_{n}^{(1)}$,

$$
\begin{aligned}
\delta E_{n}(t) & =-\alpha_{n} \mathscr{E}_{0}^{2}(t) / 4, \\
\alpha_{n} & =\frac{e^{2}}{m} \sum_{k} \frac{f_{n k}}{\omega_{n k}^{2}-\omega^{2}},
\end{aligned}
$$

where $e, m$ are the electron charge and mass, $f_{n k}$ is the oscillator strength, and $\omega_{n k}=\left(E_{n}-E_{k}\right) / \hbar$ is the transition frequency [32]. Note that the ac Stark effect for degenerate levels is more complicated and is connected to their

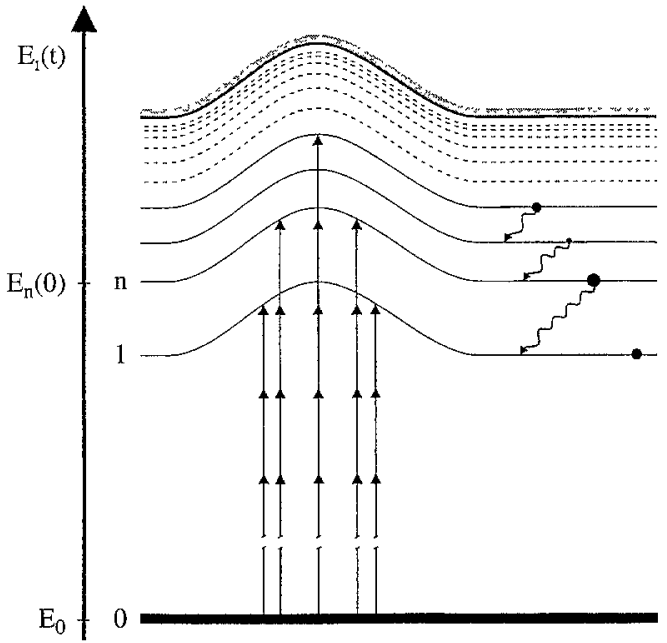

Fig. 15. Scheme of intensity-dependent multiphoton resonance excitation processes

splitting. For the ground state, the energy shift is negative $\left(\alpha_{0}>0\right)$ and usually small. For the highly excited state (taking into account that $\omega \gg\left|\omega_{n k}\right|$ and $\sum_{k} f_{n k}=1$ ) the energy shift is positive and is equal to the pondermotive potential

$\delta E_{n}(t)=U_{\mathrm{p}}(t)=e^{2} \mathscr{E}_{0}^{2}(t) / 4 m \omega^{2}$.

Therefore, the condition for the $K$-photon resonance can be written as

$K \hbar \omega=E_{n}+\delta E_{n}(t)-E_{0}-\delta E_{0} \simeq E_{n}+U_{\mathrm{p}}(t)-E_{0}$

and is demonstrated in Fig. 15. When $E_{\mathrm{i}}>U_{\mathrm{p}}>\omega$, multiphoton resonances can appear for a group of atomic transitions. For every level, the resonance occurs twice on the leading and falling edge of the laser pulse.

The time interval for these resonances is much smaller than the laser pulse duration and is defined by the first or second (in maximum) time derivative of the laser intensity. At the end of the laser pulse, a population equilibration between the ground and resonant group of levels can be produced. Therefore, neglecting coherent effects, one can assume that $N_{0} / g_{0}=N_{\mathrm{t}} / g_{\mathrm{t}}$, where $N_{\mathrm{t}}$ and $g_{\mathrm{t}}$ are the total population and statistical weight for the resonant group of levels.

The following scenario for the creation of population inversion and lasing in soft-X-ray region of spectra can be assumed. A sufficiently intense $\mathrm{KrF}$ laser pulse is focused into a low-charged preplasma and produces a population equilibration between the ground and resonant group of levels. If the preplasma electron temperature is low enough, then after the laser pulse, collisional relaxation can transfer the total population $N_{\mathrm{t}}$ to a lower lying level 1 (Fig. 15), so that $N_{1}=N_{\mathrm{t}}$. This will result in the creation of population inversion on $1-0$ transition $N_{1} / g_{1} \gg N_{0} / g_{0}$, since $g_{\mathrm{t}} \gg g_{1}$.

Investigations of the resonant multiphoton excitation as a possible pumping mechanism for soft-X-ray lasers are very appealing. Many more complicated schemes based on the resonant multiphoton pumping can be suggested. Unfortunately, at each position in the laser focus, atoms 
see a pulse of a different intensity. This complicates the experimental confirmation of the resonant multiphoton effects. Note that in experiments [7], where $\gamma \simeq 0.33$, multiphoton processes can also play an important role. Under their experimental conditions, a part of hydrogenlike Li ions could survive in excited states.

\section{Conclusion}

Experimental results on low-order nonlinear frequency up-conversion in the VUV and XUV spectral range are presented. They indicate the broad potential of shortpulse $\mathrm{KrF}$ laser systems for the efficient generation of powerful and tunable short-wavelength coherent radiation. $\mathrm{KrF}$ lasers are also interesting drivers for the realization of soft-X-ray lasers. Advantages and new possibilities are briefly discussed. $\mathrm{KrF}$ laser systems have many additional applications (in material processing, chemistry, spectroscopy, etc.), which are outside the scope of this article. Therefore, the future of the high-power short-pulse $\mathrm{KrF}$ lasers seems to be quite promising.

\section{References}

1. D.L. Matthews, P.L. Hagelstein, M.D. Rosen, M.J. Eckart, N.M. Ceglio, A.U. Hazi, H. Medecki, B.J. MacGowan, J.E. Trebes, B.L. Whitten, E.M. Campbell, C.W. Hatcher, A.M. Hawryluk, R.L. Kauffman, L.D. Pleasance, T.A. Weaver: Phys. Rev. Lett. 54, $110(1985)$

2. S. Suckewer, C.H. Skinner, H. Milchberg, C. Keane, D. Voorhees, Phys. Rev. Lett. 55, 1753 (1985)

3. J. Reintjes, C.Y. She, R.C. Eckardt, N.E. Karangelen, R.A. Andrews, R.C. Elton: Appl. Phys. Lett. 30, 480 (1977)

4. E.P. Ippen: Appl. Phys. B 58, 159 (1994)

5. C. Sauteret, G. Mainfray, G. Mourou: Laser Focus World, (1990)

6. For a review, see A.L'Huillier, L.A. Lompre, G. Mainfray, C. Manus: In Atoms in Intense Fields, ed. by M. Gavrila, Advances in Atomic, Molecular, and Optical Physics, Supplement (Academic Press, London, 1992), p. 139

7. Y. Nagata, K. Midorikawa, S. Kubodera, M. Obara, H. Tashiro, K.Toyoda: Phys. Rev. Lett. 71, 3774 (1993)

8. B.E. Lemoff, G.Y. Yin, C.L. Gordon III, C.P.J. Barty, S.E. Harris: Phys. Rev. Lett. 74, 1574 (1995)
9. B.N. Chichkov, A. Egbert, H. Eichmann, C. Momma, S. Nolte, B. Wellegehausen: Phys. Rev. A 52, 1629 (1995)

10. S. Szatmari and F.P. Schäfer: Opt. Commun. 68, 196 (1988)

11. S. Szatmari: Appl. Phys. B 58, 211 (1994)

12. P. Balcou, C. Cornaggia, A.S.L. Gomes, L.A. Lompre, A.L'Huillier: J. Phys. B: At. Mol. Opt. Phys. 25, 4467 (1992)

13. S. Szatmari and F.P. Schäfer: J. Opt. Soc. Am. B 6, 1877 (1989)

14. J.F. Reintjes, Nonlinear Optical Parametric Processes in Liquids and Gases (Academic Press, Orlando, 1984)

15. A. Tünnermann, K. Mossavi, B. Wellegehausen: Phys. Rev. A 46, 2707 (1992)

16. A. Tünnermann, C. Momma, K. Mossavi, C. Windolph, B. Wellegehausen: IEEE J. Quantum Electron. QE-29, 1233 (1993)

17. J.H. Glownia, D.R. Gnass, P.P. Sorokin: J. Opt. Soc. Am. B 11, 2427 (1994)

18. S.P. Le Blanc, Z. Qi, R. Sauerbrey: Appl. Phys. B 61, 439 (1995); S.P. Le Blanc, Z. Qi, R. Sauerbrey, Opt. Lett. 20, 312 (1995)

19. C. Momma, H. Eichmann, H. Jacobs, A. Tünnermann, H. Welling, B. Wellegehausen: Opt. Lett. 18, 516 (1993)

20. C. Momma, H. Eichmann, A. Tünnermann, P. Simon, G. Marowsky, B. Wellegehausen: Opt. Lett. 18, 1180 (1993)

21. K.Mossavi, L. Fricke, P. Liu, and B. Wellegehausen: Opt. Lett. 20, 1403 (1995)

22. J.M. Schins, P. Breger, P. Agostini, R.C. Constantinescu, H.G. Muller, A. Bouhal, G. Grillon, A. Antonetti, A. Mysyrowicz: J. Opt. Soc. Am. B 13, 197 (1996)

23. W. Theobald. C. Wülker, F.P. Schäfer, B.N. Chichkov: Opt. Commun. 120, 177 (1995)

24. S. Meyer, H. Eichmann, T. Menzel, S. Nolte, B. Wellegehausen, B.N. Chichkov, C. Momma: Phys. Rev. Lett. 76, 3336 (1996)

25. M. Steyer, F.P. Schäfer, S. Szmatmari, G. Kühnl: Appl. Phys. B 50, 265 (1990)

26. L.V. Keldysh: Zh. Eksp. Teor. Fiz. 47, 1945 (1964) [Sov. Phys. JETP 20, 1307 (1965)]

27. S. August, D. Strickland, D.D. Meyerhofer, S.L. Chin, J.H. Eberly: Phys. Rev. Lett. 63, 2212 (1989)

28. Y. Nabekawa et al.: Opt. Lett. 18, 1922 (1993)

29. R.R. Freeman, P.H. Bucksbaum, H. Milchberg, S. Darack, D. Schumacher, M.E. Geusic: Phys. Rev. Lett. 59, 1092 (1987)

30. O.L. Landen, M.D. Perry, E.M. Campbell: Phys. Rev. Lett. 59, 2558 (1987); H.G. Muller, H.B. van Linden van den Heuvell, P. Agostini, G. Petite, A. Antonetti, M. Franco, A. Migus: Phys. Rev. Lett. 60, 565 (1988); M.D. Perry, O.L. Landen: Phys. Rev. A 38, 2815 (1988); R.R. Freeman, P.H. Bucksbaum: J. Phys. B 24, $325(1991)$

31. M.P. de Boer, H.G. Muller: Phys. Rev. Lett. 68, 2747 (1992)

32. See, for example, I.I. Sobelman: Atomic Spectra and Radiative Transitions (Springer, Berlin, 1979) 